\title{
Anti-lgE: lessons from clinical trials in patients with severe allergic asthma symptomatic despite optimised therapy
}

\author{
R. Buhl
}

ABSTRACT: The efficacy of omalizumab has been extensively investigated in clinical trials in patients with severe persistent allergic (pre-treatment total immunoglobulin E 30-700 IU $\cdot \mathrm{mL}^{-1}$ ) asthma including the Investigation of Omalizumab in Severe Asthma Treatment (INNOVATE) study, which enrolled patients with inadequately controlled severe persistent allergic asthma despite receiving high-dose inhaled corticosteroid in combination with a long-acting $\beta_{2}$-agonist, and also additional controller medication if required.

In the INNOVATE study, add-on omalizumab significantly reduced clinically significant exacerbation rates by $26 \%$ ( 0.68 versus 0.91$)$, severe exacerbation rates by $50 \%(0.24$ versus 0.48 ) and emergency visit rates by $44 \%$ ( 0.24 versus 0.43$)$ and significantly improved asthmarelated quality of life (QoL) compared with placebo. In a pooled analysis of data from seven studies, add-on omalizumab significantly reduced asthma exacerbation rates by $38 \%(0.91$ versus 1.47 ) and total emergency visits by $47 \%$ (0.332 versus 0.623$)$. In addition, omalizumab significantly improved QoL versus current asthma therapy in a pooled analysis of data from six studies.

Omalizumab has demonstrated a good safety and tolerability profile in completed phase-I, -II and -III studies involving $>7,500$ patients with asthma, rhinitis or related conditions. Omalizumab represents a major advance for the treatment of severe persistent allergic asthma that is inadequately controlled despite treatment with inhaled corticosteroids and a long-acting $\boldsymbol{\beta}_{2^{-}}$ agonist.

KEYWORDS: Allergy, anti-immunoglobulin E, asthma, exacerbation, omalizumab, therapy

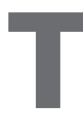
he efficacy and safety profile of omalizumab has been extensively investigated in a series of clinical studies carried out in patients with severe persistent allergic asthma. This patient group is at an increased risk of hospitalisation for exacerbations, as well as being at an increased risk of death caused by asthma. In a 28-week randomised, placebo-controlled trial, the Investigation of Omalizumab in Severe Asthma Treatment (INNOVATE) study [1] assessed the efficacy of omalizumab in patients with inadequately controlled severe persistent allergic asthma, despite receiving a high-dose inhaled corticosteroid (ICS) in combination with a long-acting $\beta_{2}$-agonist (LABA), and additional controller medication if required (Global Initiative for Asthma (GINA) 2002 step 4 therapy) [2]. Efficacy was further assessed in an analysis [3] of pooled data from seven clinical trials of omalizumab (including INNOVATE) carried out in patients with moderate-to-severe or severe asthma [1, 4-11]. Five of the seven studies were randomised, double-blind, placebo-controlled trials in which patients received omalizumab or placebo. Two studies were randomised and controlled but open label. Safety and tolerability of omalizumab across the clinical trial programme has also been analysed.

\section{THE INNOVATE STUDY}

The INNOVATE study was a randomised, placebo-controlled, double-blind study conducted in 419 patients with inadequately controlled severe persistent allergic asthma. The INNOVATE study comprised: a screening visit; an 8-week run-in phase; a 28-week double-blind treatment phase, where patients received either omalizumab or placebo by subcutaneous injection; and a 16-week follow-up phase (fig. 1). Patients included in the INNOVATE study represent the asthma population with the most severe disease and the greatest unmet medical need. All patients were receiving high-dose ICS $\left(>1,000 \mu \mathrm{g} \cdot\right.$ day $^{-1}$ beclometasone dipropionate equivalent) and a LABA, and $\sim 60 \%$ were taking additional controller medication, including oral corticosteroids $(22 \%$ of
CORRESPONDENCE

R. Buhl

Pulmonary Dept

Mainz University Hospital

Langenbeckstrasse 1

D - 55131 Mainz

Germany

Fax: 496131175545

E-mail: r.buhl@3-med.klinik.unimainz.de

STATEMENT OF INTEREST

R. Buhl has received reimbursement for attending scientific conferences and/or fees for speaking and/or consulting from Aerocrine, Altana, AstraZeneca, Bayer, Boehringer Ingelheim, GlaxoSmithKline, Novartis Pharma AG, Pfizer, Schering Plough and Zambon. R. Buhl has also received fees for research, organising education and for a member staff. The Pulmonary Dept at Mainz University Hospital received financial compensation for services performed during participation in single- and multicentre clinical phase I-IV trials organised by various pharmaceutical companies. This issue of the European Respiratory Review contains proceedings of a satellite symposium held at the 16th ERS Annual Congress, 2006, which was sponsored by Novartis Pharma AG The authors were assisted in the preparation of the text by professional medical writers at ACUMED ${ }_{\mathbb{E}}$; this support was funded by Novartis Pharma AG. 


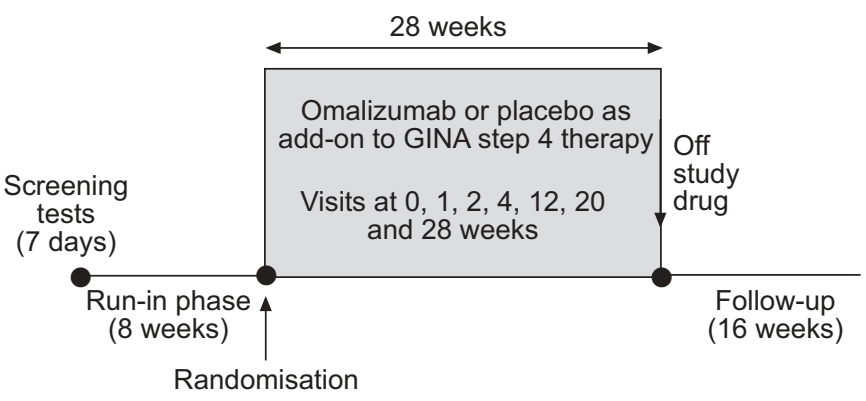

FIGURE 1. The Investigation of Omalizumab in Severe Asthma Treatment (INNOVATE) study design. GINA: Global Initiative for Asthma. Data taken from [1].

patients). Despite high levels of medication use, 67\% were considered to be at high risk of asthma-related death (previous intubation or emergency room visit/hospitalisation in the previous year), asthma had a meaningful impact on patients' quality of life (QoL) and an average of 31 school or work days had been missed in the previous year due to asthma.

The primary efficacy variable was the rate of clinically significant asthma exacerbations, defined as asthma worsening requiring treatment with systemic corticosteroids (SCS). Other outcome variables included: severe exacerbation rate (peak expiratory flow or forced expiratory volume in one second $<60 \%$ of personal best and requiring SCS); emergency visit rate (hospital admissions, emergency department and unscheduled visits to the doctor); and asthma-related QoL as assessed by the Asthma Quality of Life Questionnaire (AQLQ) [12].

In the INNOVATE trial, adding omalizumab to high-dose ICS in combination with a LABA significantly reduced the rate of clinically significant exacerbations by $26 \%$ versus add-on placebo (clinically significant exacerbation rate adjusted post hoc for an observed imbalance in exacerbation history, 0.68 versus $0.91, \mathrm{p}=0.042$; unadjusted result $19 \%$ reduction, 0.74 versus $0.92, p=0.153$; fig. 2 ). Omalizumab significantly reduced
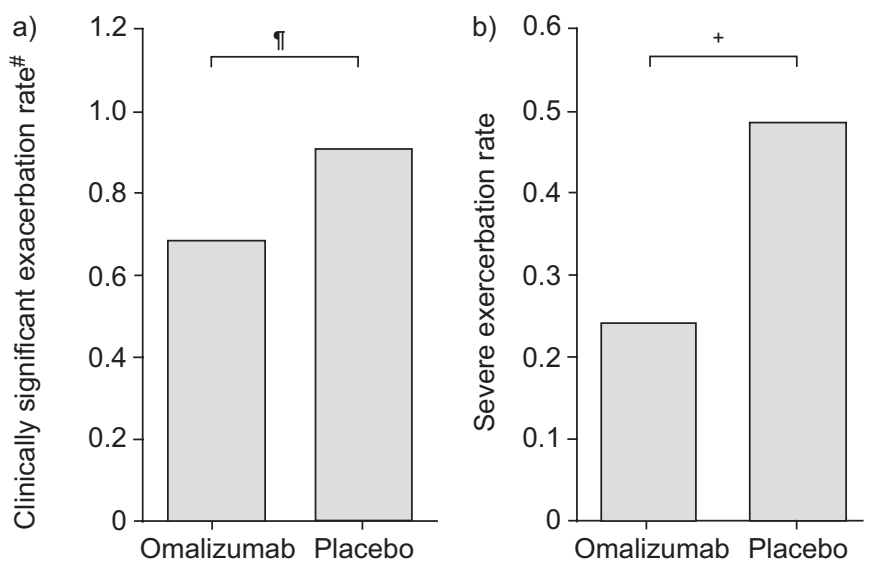

FIGURE 2. Effect of omalizumab ( $\mathrm{n}=209)$ on a) clinically significant and $b$ ) severe exacerbation rates, compared with placebo $(n=210) .{ }^{*}$ : adjustment due to a pre-study imbalance in exacerbation rate $(-19.4 \%(p=0.153)$ reduction unadjusted); ๑: $p=0.042$, exacerbation rate $26.2 \%$ lower in omalizumab group; ${ }^{+}: p=0.002$, exacerbation rate $50.0 \%$ lower in omalizumab group. Reproduced, with modifications, from [1] with permission from the publisher.

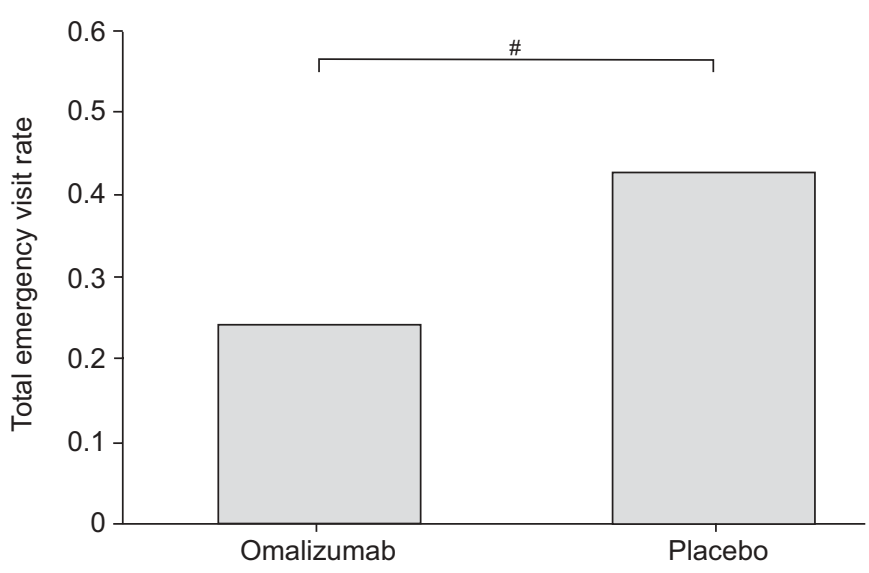

FIGURE 3. Effect of omalizumab $(n=209)$ on total emergency visit rates, compared with placebo $(n=210) .{ }^{*}: p=0.038$, visit rate $43.9 \%$ lower in omalizumab group. Data taken from [1]

severe exacerbation rate by $50 \%$ versus placebo ( 0.24 versus 0.48 , $p=0.002$; fig. 2 ) and emergency visit rate by $44 \%$ versus placebo ( 0.24 versus $0.43, p=0.038$; fig. 3 ). The number needed to treat with omalizumab in addition to GINA 2002 step 4 therapy to prevent one event per year above placebo was 2.7 for clinically significant exacerbations, 2.0 for severe exacerbations and 2.8 for total emergency visits (table 1) [13].

Importantly, add-on omalizumab treatment also provided significant QoL improvements for patients (fig. 4). Change from baseline in AQLQ overall score was 0.91 in omalizumab compared with 0.46 in the placebo group $(p<0.001)$. Significant $(p<0.01)$ improvements were seen in all individual domains. Significantly more patients taking omalizumab achieved clinically meaningful improvements in QoL compared with placebo ( 60.8 versus $47.8 \%$; $p=0.008$ ), equating to $a \geqslant 0.5$-point improvement from baseline in AQLQ overall score measured across the domains of activity limitations, asthma symptoms, emotional function and environmental exposure.

\section{POOLED ANALYSIS}

Efficacy was further assessed in an analysis of pooled data from seven clinical trials of omalizumab (including INNOVATE) carried out in patients with asthma [3]. The pooled analysis included 4,308 patients (2,511 patients received omalizumab), of which $93 \%$ had severe persistent asthma. Adding omalizumab to current asthma therapy in the larger pooled population from all seven studies consistently

TABLE 1 Number needed to treat (NNT) with omalizumab in addition to step 4 therapy to prevent one event per year above placebo

Efficacy outcome
N $^{\#} \quad$ NNT

$\begin{array}{ll}\text { Clinically significant exacerbation } & 2.7 \\ \text { Severe exacerbation } & 2.0 \\ \text { Emergency visit } & 2.8\end{array}$

\#: note that these are not all separate events. Data taken from [13] 


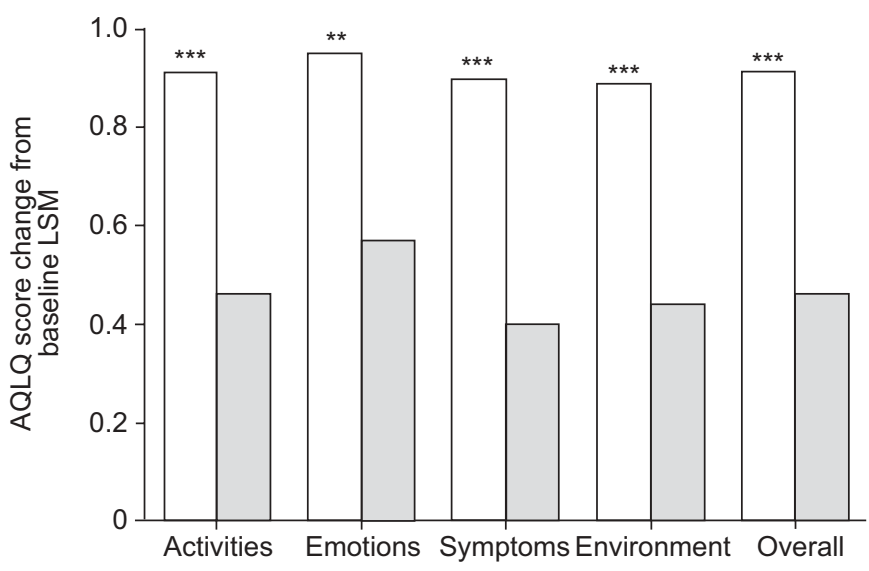

FIGURE 4. Effect of omalizumab $(\square)$ versus placebo $(\square)$ on quality of life AQLQ: Asthma Quality of Life Questionnaire; LSM: least squares mean. **: $p<0.01$; $* * *: p<0.001$. Data taken from [1].

confirmed the findings seen in INNOVATE. Add-on omalizumab significantly reduced asthma exacerbation rates by $38.3 \%$ versus control (0.91 versus 1.47; $\mathrm{p}<0.0001$; table 2 ) and total emergency visits by $47 \%$ versus control (0.332 versus 0.623 ; $\mathrm{p}<0.0001$; table 3$)$. In the pooled analysis, omalizumab significantly reduced each component of the composite total emergency visit rate. Hospital admissions were reduced by $51 \%(p=0.041)$, emergency room visits by $60 \%(p=0.013)$ and unscheduled doctor visits by $43 \%(\mathrm{p}=0.0003$; table 3$)$.

In the pooled analysis, QoL was measured in five clinical trials in addition to INNOVATE, with 1,221 patients receiving omalizumab and 1,032 receiving placebo/control across the six studies [14]. Omalizumab consistently improved QoL, delivering significantly greater improvements in the AQLQ overall score compared with placebo/control (1.01 versus 0.61 ; $p<0.001)$ and in the proportion of patients in the pooled population recording $a \geqslant 0.5$-point improvement in AQLQ overall score ( $66.3 \%$ omalizumab versus $52.4 \%$ placebo/control; $\mathrm{p}<0.001)$.

\section{SAFETY AND TOLERABILITY}

The safety and tolerability profile of omalizumab was evaluated in an analysis of data from completed phase-I, -II and -III studies involving $>7,500$ adult and adolescent patients with asthma, rhinitis or related conditions [15]. Of these

\begin{tabular}{|c|c|c|c|}
\hline Study/first author [Ref.] & $\begin{array}{c}\text { Annual exacerbation } \\
\text { rate treatment } \\
\text { difference }\end{array}$ & Reduction \% & $p$-value \\
\hline INNOVATE study [1] & 0.49 & $26.2^{\#}$ & $0.042^{\#}$ \\
\hline ETOPA study [10] & 1.49 & 60.4 & $<0.001$ \\
\hline SOLAR study [8] & 0.29 & 37.5 & 0.027 \\
\hline Busse [7] & 0.40 & 40.3 & $<0.001$ \\
\hline SOLÈR [4] & 0.70 & 57.6 & $<0.001$ \\
\hline Holgate [9] & 0.42 & 26.5 & 0.165 \\
\hline ALTO study [11] & 0.18 & 15.3 & 0.077 \\
\hline Pooled [3] & 0.56 & 38.3 & $<0.0001$ \\
\hline
\end{tabular}

patients, $>5,300$ received omalizumab, including 3,700 with moderate-to-severe persistent asthma.

In all controlled studies (omalizumab: $n=3,678$; control: $\mathrm{n}=2,452$ ), adverse events (AEs) were reported in $74.8 \%$ of patients receiving omalizumab and in $75.2 \%$ of patients in the control group. Serious AEs (i.e. events that were fatal or life threatening, required hospitalisation, were significantly or permanently disabling or incapacitating, constituted a congenital anomaly or a birth defect, or were considered medically significant) were reported by $4.2 \%$ of patients receiving omalizumab and by $3.8 \%$ of patients in the control group. Nasopharyngitis, upper respiratory tract infection, headache and sinusitis were the most frequently reported AEs in the omalizumab and control groups (table 4). Any differences observed between the groups were small and not indicative of specific organ toxicity. AEs were generally of mild-tomoderate severity and of short duration. Severe AEs occurred more frequently in the control group than in the omalizumab group (12.6 versus 10.8\%; table 5). Serious AEs occurred infrequently (omalizumab 4.5\%, control 3.8\%). Few patients discontinued due to AEs (omalizumab 2\%, control 1\%). In placebo-controlled allergic asthma studies, overall incidence of suspected drug-related AEs was similar in the omalizumab and placebo groups (9.2 versus $7.6 \%$, respectively). Post-launch data in the USA $(\sim 40,000$ patients $)$ are consistent with omalizumab clinical trials data.

TABLE 3 Effect of omalizumab on emergency visits for asthma

\begin{tabular}{cc}
\multicolumn{2}{c}{ Rate per year } \\
\hline Omalizumab $\quad$ Control
\end{tabular}

\begin{tabular}{|c|c|c|c|c|}
\hline Total emergency visits & 0.332 & 0.623 & $<0.0001$ & 47 \\
\hline Hospital admissions & 0.030 & 0.062 & 0.041 & 51 \\
\hline Emergency room visits & 0.026 & 0.066 & 0.013 & 60 \\
\hline Unscheduled doctor visits & 0.252 & 0.443 & 0.0003 & 43 \\
\hline
\end{tabular}

Data taken from [3]. 


\begin{tabular}{lcc}
\hline TABLE 4 & $\begin{array}{l}\text { Most common adverse events } \\
\text { studies }\end{array}$ & in all controlled \\
\hline Adverse event & Omalizumab & Control \\
\hline Subjects n & 3678 & 2452 \\
Any adverse event & 74.8 & 75.2 \\
Upper respiratory tract infection & 15.7 & 15.7 \\
Headache & 15.5 & 15.6 \\
Nasopharyngitis & 14.4 & 15.9 \\
Sinusitis & 10.1 & 12.0 \\
Pharyngolaryngeal pain & 6.4 & 6.0 \\
Cough & 5.9 & 7.1 \\
Bronchitis & 5.2 & 5.6 \\
Influenza & 5.0 & 6.6 \\
\hline
\end{tabular}

Data are presented as $\%$, unless otherwise stated. ${ }^{*}: \geqslant 5 \%$ in any group.

\section{ADVERSE EVENTS OF SPECIAL INTEREST}

The risk of specific hypothetical safety concerns that might be associated with a monoclonal antibody targeting an immune system mediator (e.g. hypersensitivity reactions, malignant neoplasia and parasitic infections) as well as regarding issues that were identified during pre-clinical investigation (thrombocytopenia) have also been assessed.

\section{Hypersensitivity}

As omalizumab is a protein, it might be expected to be associated with hypersensitivity reactions and related immunological effects. As residues of murine origin constitute $<5 \%$ of the omalizumab molecule and omalizumab cannot crosslink high-affinity immunoglobulin (Ig)E receptors (FceRI) and activate effector cells, omalizumab has low anaphylactogenic potential. None of the omalizumab-treated patients developed measurable anti-omalizumab antibodies. Skin rash (urticaria) is a common manifestation of allergic reactions. In phase-II and -III trials, the incidence of urticaria was similar in patients treated with omalizumab $(1.1 \%)$ and control patients $(1.2 \%)$, and the incidence of severe systemic hypersensitivity was $0.1 \%$ in both groups. As omalizumab is an Ig designed to bind to the patient's IgE, there is a theoretical possibility of immunecomplex-mediated AEs following administration. There has

\begin{tabular}{lcc}
$\begin{array}{l}\text { TABLE } 5 \\
\text { Most common severe adverse events } \\
\text { controlled in all }\end{array}$ & \\
Adverse event & Omalizumab & Control \\
\hline Subjects $\mathbf{n}$ & 3678 & 2452 \\
Any severe adverse event & 10.8 & 12.6 \\
Headache & 1.5 & 1.6 \\
Upper respiratory tract infection & 0.7 & 0.6 \\
Sinusitis & 0.5 & 1.0 \\
Influenza & 0.3 & 0.7 \\
Nasopharyngitis & 0.2 & 0.5 \\
\hline
\end{tabular}

Data are presented as $\%$, unless otherwise stated. ${ }^{*}: \geqslant 0.5 \%$ in any group. been no incidence of immune-complex disease in clinical trials with omalizumab.

Based on spontaneous reports and an estimated exposure of 57,300 patients from June 2003 to December 2006 (USA), the frequency of anaphylaxis attributed to omalizumab use was estimated to be $\geqslant 0.2 \%$ of patients (United States Prescribing Information, July 2007). As a precaution, medications for the treatment of anaphylactic reactions should be available for immediate use following administration of omalizumab (European Union (EU) summary of product characteristics (SmPC)).

\section{Malignancy}

During the omalizumab clinical programme, a number of cancer cases were reported with a slight numerical imbalance between those arising in the omalizumab-treated group (25 cases; $0.5 \%$ ) compared with the control group (5 cases; $0.18 \%$ ). No cases of malignant neoplasia were considered to be drugrelated by a panel of independent oncologists blinded to treatment assignment. An in-depth clinical and statistical analysis of malignant neoplasia reported in multiple-dose clinical studies of omalizumab of up to 4 yrs duration was conducted [16]. In addition, a literature review and a comparison of cancer rates with the National Institutes of Health (NIH) Surveillance, Epidemiology, and End Results (SEER) database were undertaken.

The malignancy rate per 1,000 patient-yrs in the omalizumab group was comparable with the control group $(\mathrm{p}>0.5)$. The range of relative risk estimates across omalizumab phase I-III clinical trials (0.75-5.14) was consistent with estimates for asthmatic populations reported in the literature (0.75-2.1). A meta-analysis of five cohort studies in asthmatic populations [17-21] found no association between asthma and cancer rates (incidence ratio (95\% confidence interval) 0.95 (0.69-1.30)), suggesting that asthma is neither a risk factor nor a protective factor for cancer. A comparison of cancer rates (from the clinical programme) with the NIH SEER database found that the standardised incidence ratio (SIR) of observed/expected number of events (malignant neoplasia) in the omalizumab group was 0.99 (0.55-1.63), i.e. near identical to that expected in the general population, whereas the control group SIR was one-third of that expected $(0.31(0.04-1.11))$. Over time and with increased exposure (sample size and duration) the SIR for the omalizumab-treated group has decreased from the 1.3 (0.69-2.33) value at the time of Food and Drug Administration submission in June 2003.

Overall, clinical data do not suggest a causal link between omalizumab and cancer. A 5-yr post-marketing surveillance study (the EXCELS study: an epidemiological study of Xolair ${ }^{\circledR}$ (omalizumab): evaluating clinical effectiveness and long-term safety in patients with moderate-to-severe asthma) involving 7,500 patients is ongoing.

\section{Helminth infection}

Parasitic helminth infection induces production of IgE, which is regarded as an important immune effector mechanism against helminth infection. There is, therefore, a theoretical possibility that treatment with omalizumab could increase susceptibility to helminth infection. The risk of parasitic 
infection was investigated in a 52-week, randomised, doubleblind, placebo-controlled study in 137 adults and adolescents with allergic asthma or allergic rhinitis at high risk of intestinal helminth infection [22]. The trial showed a slight numerical increase in infection incidence with omalizumab (50 versus $41 \%$ ) that was not statistically significant. Importantly, course, severity and clinical outcome were unaltered. Caution may be warranted in patients at high risk of helminth infection, in particular when travelling to areas where helminthic infections are endemic (EU SmPC).

\section{Thrombocytopenia}

Reduced platelet counts were observed in juvenile cynomolgus monkeys receiving omalizumab at doses 3.7-20-fold greater than the highest dose recommended in humans. This observation was made after initiation of the phase-III clinical trials and led to the implementation of increased surveillance of platelet counts in all ongoing and subsequent clinical studies. In all completed studies, there was no evidence of a clinically relevant impact on platelet count during omalizumab treatment. Additional platelet testing is not a requirement of the USA or EU label for omalizumab.

\section{CONCLUSIONS}

The data derived from the extensive omalizumab clinical trial programme indicate that add-on omalizumab is effective (reducing exacerbations, emergency visits and hospitalisations, and improving quality of life) and well tolerated. Omalizumab addresses an unmet medical need in patients with severe persistent allergic asthma whose symptoms persist despite receiving high-dose inhaled corticosteroids and long-acting $\beta_{2^{-}}$ agonists, allowing these patients to live beyond the limitations of their asthma.

\section{REFERENCES}

1 Humbert M, Beasley R, Ayres J, et al. Benefits of omalizumab as add-on therapy in patients with severe persistent asthma who are inadequately controlled despite best available therapy (GINA 2002 step 4 treatment): INNOVATE. Allergy 2005; 60: 309-316.

2 Global Initiative for Asthma. Global strategy for asthma management and prevention. NIH Publication No 02-3659. Bethesda, National Institutes of Health/National Heart, Lung, and Blood Institute, 2005.

3 Bousquet J, Cabrera P, Berkman N, et al. The effect of treatment with omalizumab, an anti-IgE antibody, on asthma exacerbations and emergency medical visits in patients with severe persistent asthma. Allergy 2005; 60: 302-308.

4 Solèr M, Matz J, Townley R, et al. The anti-IgE antibody omalizumab reduces exacerbations and steroid requirement in allergic asthmatics. Eur Respir J 2001; 18: 254-261.

5 Lanier BQ, Corren J, Lumry W, Liu J, Fowler-Taylor A, Gupta N. Omalizumab is effective in the long-term control of severe allergic asthma. Ann Allergy Asthma Immunol 2003; 91: 154-159.

6 Buhl R, Solèr M, Matz J, et al. Omalizumab provides longterm control in patients with moderate-to-severe allergic asthma. Eur Respir J 2002; 20: 73-78.

7 Busse W, Corren J, Lanier BQ, et al. Omalizumab, anti-IgE recombinant humanized monoclonal antibody, for the treatment of severe allergic asthma. I Allergy Clin Immunol 2001; 108: 184-190.

8 Vignola AM, Humbert M, Bousquet J, et al. Efficacy and tolerability of anti-immunoglobulin E therapy with omalizumab in patients with concomitant allergic asthma and persistent allergic rhinitis: SOLAR. Allergy 2004; 59: 709-717.

9 Holgate ST, Chuchalin AG, Hébert J, et al. Efficacy and safety of a recombinant anti-immunoglobulin E antibody (omalizumab) in severe allergic asthma. Clin Exp Allergy 2004; 34: 632-638.

10 Ayres JG, Higgins B, Chilvers ER, Ayre G, Blogg M, Fox H. Efficacy and tolerability of anti-immunoglobulin E therapy with omalizumab in patients with poorly controlled (moderate-to-severe) allergic asthma. Allergy 2004; 59: 701-708.

11 Genentech, Inc. A multicenter, randomized, controlled, open-label study to evaluate the safety of $\mathrm{Xolair}^{\mathrm{TM}}$ in moderate to severe persistent asthma subjects already treated with other therapies (ALTO). http://clinicalstudyresults.gene.com/q2143g.pdf Date last updated: November 2002. Date last accessed: May 2007.

12 Juniper EF, Guyatt GH, Epstein RS, Ferrie PJ, Jaeschke R, Hiller TK. Evaluation of impairment of health related quality of life in asthma: development of a questionnaire for use in clinical trials. Thorax 1992; 47: 76-83.

13 Humbert M, Ayre G, Fox H, Blogg M. Number needed to treat to prevent one medically significant event per year with add-on omalizumab: INNOVATE. XXV EAACI Congress (Vienna, 10-14 June 2006) Abstract Book, 2006; p.41, 125.

14 Chipps B, Buhl R, Beeh KM, Fox H, Thomas K, Reisner C. Improvement in quality of life with omalizumab in patients with severe allergic asthma. Curr Med Res Opin 2006; 22: 2201-2208.

15 Corren J, Casale T, Lanier BQ, Blogg M, Reisner C, Gupta N. Omalizumab is well tolerated in adolescent/ adult patients ( $\geqslant 12$ years) with moderate-to-severe persistent asthma. J Allergy Clin Immunol 2005; 115: S75.

16 Fernández C, Busse W, Reisner C, Gupta N. Clinical data do not suggest a causal relationship between omalizumab therapy and cancer. Proc Am Thorac Soc 2005; 2: A359.

17 Eriksson NE, Holmén A, Högstedt B, Mikoczy Z, Hagmar L. A prospective study of cancer incidence in a cohort examined for allergy. Allergy 1995; 50: 718-722.

18 Källén B, Gunnarskog J, Conradson TB. Cancer risk in asthmatic subjects selected from hospital discharge registry. Eur Respir J 1993; 6: 694-697.

19 McWhorter WP. Allergy and risk of cancer. A prospective study using NHANESI followup data. Cancer 1988; 62: 451-455.

20 Mills PK, Beeson WL, Fraser GE, Phillips RL. Allergy and cancer: organ site-specific results from the Adventist Health Study. Am J Epidemiol 1992; 136: 287-295.

21 Vesterinen E, Pukkala E, Timonen T, Aromaa A. Cancer incidence among 78,000 asthmatic patients. Int J Epidemiol 1993; 22: 976-982.

22 Cruz AA, Lima F, Sarinho E, et al. Safety of antiimmunoglobulin E therapy with omalizumab in allergic patients at risk of geohelminth infection. Clin Exp Allergy 2007; 37: 197-207. 